\title{
Spatial features of non-thermal SZ effect in galaxy clusters
}

\author{
E. Palladino*, S. Colafrancesco ${ }^{\dagger}$ and P. Marchegiani ${ }^{\dagger}$ \\ *Università "La Sapienza", P.le Aldo Moro 5, Rome, Italy \\ † Osservatorio Astronomico di Roma, Monteporzio, Italy
}

\begin{abstract}
We investigate the spatial behaviour of the total comptonization parameter $y_{\text {tot }}$ evaluated for a galaxy cluster containing two population of electrons: the thermal population, with energy around some KeV and whose trace is evident in the X-ray emission of the ICM (Intra-Cluster Medium), and the relativistic population, which give rise to the radio halo emission found in several clusters of galaxies. We present the first results obtained from our analysis showing that there are remarkable features in such spatial trend, which might throw a new light in understanding the cluster internal processes.
\end{abstract}

Recently has been investigated the possibility that the thermal SZ effect, i.e. the shift in photon energy of the cosmic microwave background radiation (CMBR) due to its passage through the intra-cluster medium (ICM) ([1]], [2], [3]), could be implemented by the so called non-thermal effect due to a relativistic electron population with energy of order tens of $\mathrm{KeV}$, instead of the usual thermal amount of some $\mathrm{KeV}$ ([8], [\$]).

Here we stress the fact that the presence of a non-thermal SZ effect also produces spatial variations of the total SZ radial profile of the cluster.

Let us consider a galaxy cluster in which are present two kind of electron populations: the first one is thermal, i.e. it follows a relativistic maxwellian velocity distribution, the second one is non thermal, i.e. its energy spectrum is relativistic in a certain range. In particular we have used a phenomenological complex spectrum (double-power law) with slopes $\alpha_{r} \sim 2.5$ at $E>E_{*}$ and $\alpha_{x} \sim-0.5$ at $E<E_{*}$ with the break set at $E_{*} \sim 200 \div 400 \mathrm{MeV}$, suitable to reproduce the whole set of non thermal phenomena found in several galaxy clusters [6]. Consequently, the behaviour of this second population can not be treated with the Kompaneets approach usually used to describe the dynamic of the thermal one (for further details on a complete relativistic treatment of the non-thermal effect see [7]) . In a first approximation we consider these populations independently superposed, so it is possible to completely separate their contribution to the total SZ effect on the incoming photons of the CMBR (Colafrancesco, Marchegiani \& Palladino, these Proceedings, for more details).

The inverse Compton scattering of the CMB photons by the electrons of the ICM is theoretically described by the so-called comptonization parameter. For a thermal radiation field and in the more general form such a contribution can be expressed as $y_{t h}=y_{0, t h} \cdot g(x)$, where $y_{0, t h}$ physically represents the time spent by the radiation in the medium and it is proportional to the integral of the thermal electron kinetic pressure $P_{t h}=n_{e} k_{B} T_{e}$, while the function $g(x)$ contains the dependence of the effect from the a-dimensional frequency $x=h \mathrm{v} / k_{B} T_{C M B}$ of the background radiation.

According to the classical approach we can describe the kinetic pressure $P_{t h}$ using a theoretical model in which we assume known the spatial distribution and the temperature of the electron population. The model we have used is the isothermal $\beta$-model [9], according to which the temperature of the ICM is constant and equal to $T_{e}$ and the electron density $n_{e}$ has a spherical distribution parameterized by the relation

$$
n_{e}(\mathbf{r})=n_{e 0}\left[1+\left(\frac{r}{r_{c}}\right)^{2}\right]^{-3 \beta / 2}
$$

where $n_{e 0}$ is the electron density at the center of the cluster, $r_{c}$ is the core-radius and $\beta=\mu m_{p} v^{2} / k_{B} T_{e}$ is the exponent observed to be in the range $0.6-1$ (see, e.g., [ $₫$ ). The detailed values of such parameters are found from the analysis of X-ray emission maps produced by the thermal electrons of the cluster. 
TABLE 1. Values of the parameters used for the COMA cluster study

\begin{tabular}{rrrrrrrrr}
\hline$z$ & $\begin{array}{r}k_{B} T_{e} \\
(\mathrm{keV})\end{array}$ & $\begin{array}{r}r_{c, X} \\
\left(h_{50}^{-1} \mathrm{Mpc}\right)\end{array}$ & $\begin{array}{r}r_{c, \text { rad }} \\
\left(h_{50}^{-1} \mathrm{Mpc}\right)\end{array}$ & $\begin{array}{r}r_{\text {lim }, X} \\
\left(h_{50}^{-1} \mathrm{Mpc}\right)\end{array}$ & $\begin{array}{r}r_{\text {lim }, \text { rad }} \\
\left(h_{50}^{-1} \mathrm{Mpc}\right)\end{array}$ & $\beta_{X}$ & $\beta_{\text {rad }}$ & $\begin{array}{r}n_{e 0, X} \\
\left(h_{50}^{2} \mathrm{~cm}^{-3}\right)\end{array}$ \\
\hline 0.0232 & 8.5 & 0.42 & 0.4 & 4.2 & 1.25 & 0.75 & 0.8 & $3.0 \cdot 10^{-3}$ \\
\hline
\end{tabular}

Using the model (11), calling $P_{t h}^{0}=n_{e 0} k_{B} T_{e}$ the central kinetic pressure and calculating the integral along the line of sight [ [ 4 , we obtain the final expression for the thermal comptonization parameter:

$$
y_{0, t h}=\frac{\sigma_{T}}{m_{e} c^{2}} P_{t h}^{0} r_{c} Y_{t h}\left(\theta, \theta_{c}\right) .
$$

In the function $Y_{t h}$, whose explicit expression is given by

$$
Y_{t h}\left(\theta, \theta_{c}\right)=\sqrt{\pi} \frac{\Gamma\left(\frac{3}{2} \beta-\frac{1}{2}\right)}{\Gamma\left(\frac{3}{2} \beta\right)}\left[1+\left(\frac{\theta}{\theta_{c}}\right)^{2}\right]^{\frac{1}{2}-\frac{3}{2} \beta},
$$

is contained the dependence of the effect from the spatial coordinates, i.e. from the angle $\theta$ between the center of the cluster and the direction of observation, while $\theta_{c}=r_{c} / D_{A}$ is the angular core radius as deduced from the X-ray data. The angular diameter distance, $D_{A}$, of the cluster, given in terms of the redshift, contains all the information relative to the assumed cosmological model; in all our work we have put null the cosmological constant and the curvature and the Hubble constant is in the form $H_{0}=h_{50} 50 \mathrm{~km} \mathrm{sec}^{-1} \mathrm{Mpc}^{-1}$, with $h_{50}=1$.

Taking into account that we use the same formalism to describe both the thermal and non thermal contributions to the total effect, we can soon write down all the expressions we have previously obtained also in the case of the relativistic population, in which we again parameterized the electron density with a spherical distribution, i.e. with a $\beta$-model. One fundamental aspect to underline is the difference between the relativistic distribution and the thermal one, which consists in the fact that the spherical radius of the first distribution must be truncated at some limiting radius $r_{l i m}$, as it results from the measurements of the radio emission from clusters of galaxies, that in the case for example of COMA is found to be $r_{\text {lim }}=1.25 h_{50}^{-1} \mathrm{Mpc}$.

So for the non thermal comptonization parameter we have:

$$
y_{0, \text { non-th }}=\frac{\sigma_{T}}{m_{e} c^{2}} P_{t h}^{0} r_{c, \text { rad }} \bar{P}_{0} Y_{\text {non-th }}\left(\theta, \theta_{c, r a d}\right),
$$

where the subscript " $r a d$ " is used to remember that these quantities refer to the relativistic electrons that emits in the radio band of the electromagnetic spectrum (they in general belong to the radio halo of the cluster), while $\bar{P}_{0}=P_{r e l}^{0} / P_{t h}^{0}$ is the ratio between the relativistic and the kinetic pressures of the electrons in the centre of the cluster. The function $Y_{\text {non-th }}\left(\theta, \theta_{c, \text { rad }}\right)$, in which $\theta_{c, \text { rad }}=r_{c, \text { rad }} / D_{A}$ is the angular core radius of the radio halo, has the same expression as the one of eq. (3), in which we substitute the parameters proper of the non-thermal effect.

Now we have all the necessary information to put together the two previous contributions in a whole expression for the total comptonization parameter $y_{t o t}$ given by the sum $y_{t h}+y_{n o n-t h}$. Elaborating this expression we have the final relation used to obtain our results (here the subscript " $X$ " refers to the thermal quantities given by the clusters $X$-ray maps):

$$
y_{t o t}=\frac{\sigma_{T}}{m_{e} c^{2}} P_{t h}^{0}\left\{r_{c, X} Y_{t h}\left(\theta, \theta_{c, X}\right) \cdot g(x)+r_{c, r a d} \bar{P}_{0} Y_{n o n-t h}\left(\theta, \theta_{c, r a d}\right) \cdot \tilde{g}(x)\right\} .
$$

In Tab.1 we present the parameters used to make the analysis and to obtain the results we present for the COMA cluster.

In Fig. 1 we show the behaviour of the comptonization parameter, where we have fixed four values of the adimensional frequency $x$ (see Tab.2), to simulate the frequency channels in a common observational experiment devoted to measure clusters properties at millimeter and sub-millimeter wavelength and three values of the ratio $\bar{P}_{0}$ between the thermal and relativistic pressures of the electron populations, i.e. $0.05,0.49,1.48$. In each panel we graph the spatial trend of the total SZ effect comptonization parameter $y_{t o t}$ together with the thermal one $y_{t h}$ founded for the same physical values, to make a comparison among the different curves, from the minimum of the effect $\left(x_{\min } \sim 2.5\right)$, the zero $\left(x_{0} \sim 3.8\right)$, to its maximum $\left(x_{\max } \sim 6.2\right)$. 
TABLE 2. The four values of $x$ chosen to make the analysis (see text for details).

\begin{tabular}{cccc}
\hline Channel & $\lambda(\mathbf{m m})$ & $v(\mathbf{G H z})$ & $x$ \\
\hline 1 & 2.1 & 142.857 & 2.51 \\
2 & 1.4 & 214.286 & 3.77 \\
3 & 1.1 & 272.727 & 4.80 \\
4 & 0.85 & 352.941 & 6.21 \\
\hline
\end{tabular}

As one can see, the detection of non-thermal effect is greater in the first two channels, even if the second channel presents a difficult in measuring the effect for the presence of the maximum of the kinetic one that can be in principle greater then the non-thermal one. Going towards higher frequencies $(x>5)$, we find that there is no observational evidence of the non-thermal effect with respect to the thermal one.

Another point is the jump one can see in both the upper panels: it is due to the fact that the relativistic population has a core radius smaller than the optical radius of the cluster (that is given by $r_{c, X}$ ). The possibility to detect such a jump in the spatial distribution is linked to the angular resolution of the experiment devoted to measure the effect: it is possible, in fact, that a window function of many arc-min, with respect to the angular dimension of the examined cluster, cannot see such a characteristic spatial feature, for the signal has to be convoluted with the instrumental response.

In conclusion we underline the importance of specific spectral and spatial features of the non-thermal SZ effect that can be detected through a multi-frequency observation with narrow-band detectors: the best observational strategy is to check the frequency range $x \sim 2 \div 8$ where the spectral features allow to distinguish the non-thermal effect to the thermal one.

This is very important since the SZ effect is a remarkable tool for cosmology (i.e. with the measurement of the Hubble constant $H_{0}$ ) and for better knowing the astrophysics of clusters. We stress that the PLANK surveyor experiment has the possibility to detect the total SZ effect (thermal and non-thermal one) in a a large number of nearby radio-halo (with a relativistic electron component) clusters.

\section{REFERENCES}

1. Sunyaev, R.A. and Zel'dovich, Ya.B. 1972, Comments Astrophys. Space Sci., 4, 173

2. Rephaeli, Y. 1995, ARA\&A, 33, 541

3. Birkinshaw, M. 1999, Physics Report, 310, 97

4. Sarazin, C.L. 1988, 'X-ray emission from clusters of galaxies', Cambridge University Press

5. Blasi, P. Stebbins, A. and Olinto, A. 2000, ApJ. 535. L71

6. Petrosian, V. 2001, ApJ, in press (preprint astro-ph/0101145)

7. Colafrancesco, S., Marchegiani, P. and Palladino, E. 2001, A\&A, submitted

8. Ensslin, T. and Kaiser, C. 2000, A\&A, 360, 417

9. Cavaliere, A. and Fusco-Femiano, R. 1976, A\&A, 49, 137 

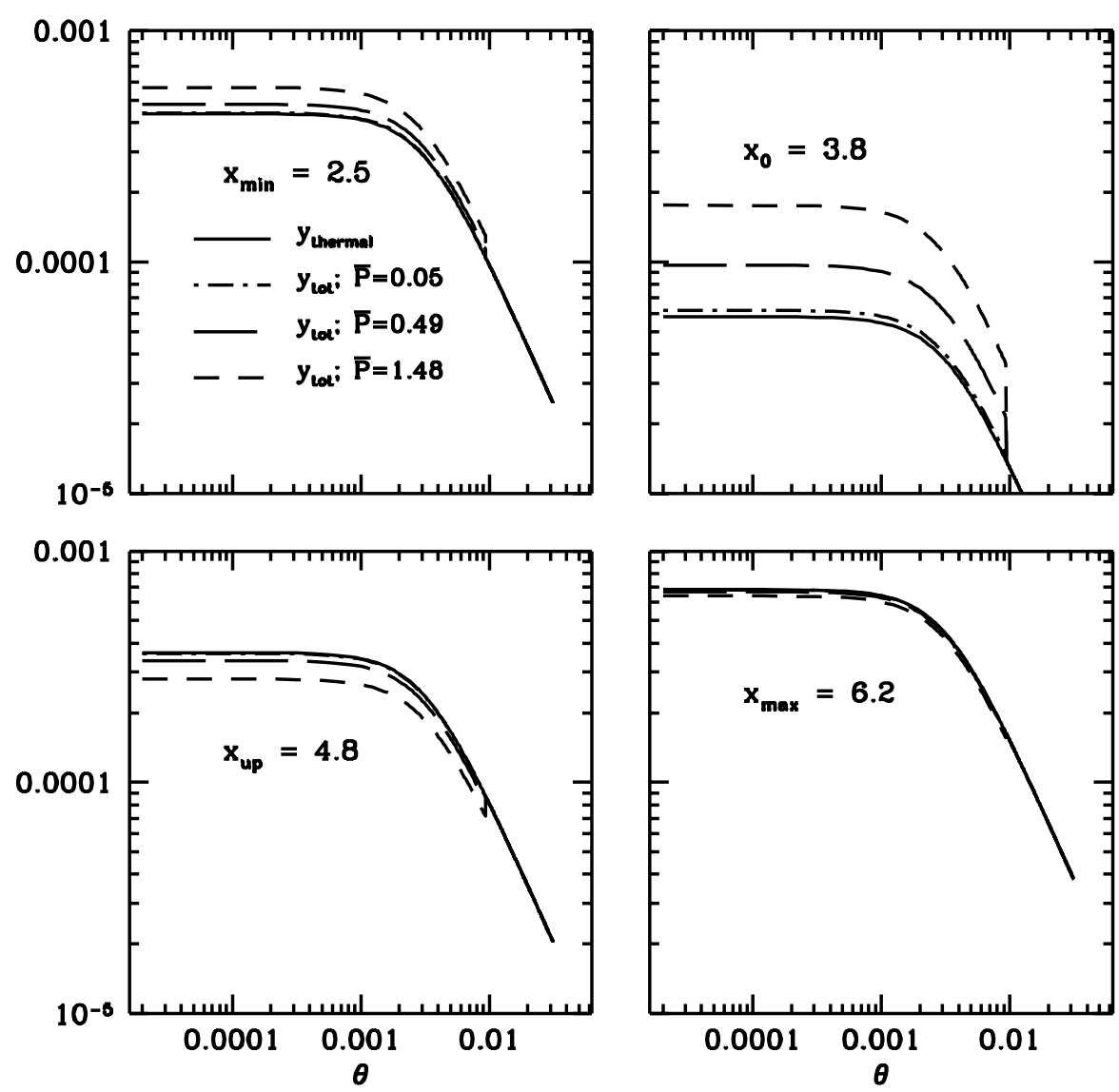

FIGURE 1. The spatial dependence of the total SZ effect produced by the combination of the thermal and relativistic electrons in COMA, with respect to the thermal one (thin line). The four panels refers to the four values of the frequency $x$ and in each one there are plotted the case for $\bar{P}_{0}=0.05$ (point-dash line), for $\bar{P}_{0}=0.49$ (long-dash line) and $\bar{P}_{0}=1.48$ (short-dash line). 